\title{
EL ESTILO COGNITIVO Y SU INFLUENCIA EN LA SOLUCIÓN DE PROBLEMAS EN QUIMICA. Un estudio en el aula de clase
}

\author{
Myriam Esperanza Sandoval Q. \\ Inem Tunja. \\ Humberto Caicedo López \\ Universidad Pedagógica Nacional.
}

\begin{abstract}
The experimental study was made to get additional empirical evidence to show how the cognitive style in its field dependence-independence dimension differentiate students with different aptitude to solve chemistry problems. Twenty eight students participated, 11 female and 17 male, average age seventeen. The test used was a set of simple problems, to obtain information about level of perception and selected strategy to get an appropriate solution.
\end{abstract}

\section{RESUMEN}

El estudio busca obtener evidencia empírica adicional que permita sostener que el estilo cognitivo en las dimensiones dependencia-independencia de campo permite diferenciar personas con mayor o menor aptitud para la solución de problemas en química. El trabajo se hizo con 28 estudiantes de diferente sexo y edad promedio de 17 años. Se utilizaron problemas sencillos en los que se buscaba obtener información sobre grado de percepción del problema y estrategia utilizada para obtener la solución.

\section{INTRODUCCION}

Una de las principales dificultades que enfrentan los estudiantes en las asignaturas de química, física y matemáticas y en general en las ciencias, es la solución de problemas, entendidos estos, no como ejercicios en los que solo es necesario aplicar unas fórmulas o utilizar un algoritmo que tiene disponible en forma inmediata el que resuelve, si no como aquellas situaciones en que la solución no es evidente, en las que existe un vacío entre la situación que plantea el enunciado del problema y la respuesta que se quiere alcanzar.

La solución de verdaderos problemas de contenido científico constituye una tarea intelectualmente compleja, que supone el empleo de conocimiento tanto declarativo como procedimental, en la que abundan los aspectos no verbales y con frecuencia la 
representación figurativa del problema resulta crucial (Witkin, 1985). Existe además, una etapa en la solución de problemas en la que es necesario desenmascarar o aislar de un contexto dado la información relevante, manejar simultáneamente los diferentes elementos del problema en tal forma que desemboque en una reestructuración que transforme el enunciado en algo mas comprensible (Bodner, 1986). Por otra parte se ha demostrado que factores o trastornos emocionales, a las que son más sensibles individuos con características bien definidas de funcionamiento cognitivo, tienen repercusión en la solución de tareas intelectuales como las que ocurren en la solución de problemas en condiciones de examen. Estas y otras razones han hecho que los investigadores consideren con mas atención la influencia del estilo cognitivo, en la solución de problemas y en general en el proceso de enseñanza de las ciencias.

El termino estilo cognitivo que se maneja en el estudio se refiere manifestación de la manera de ser, del carácter de cada persona en cuanto a la forma característica como se relaciona con el ambiente que lo rodea. Se considera que el estilo cognitivo es la etapa final de un proceso evolutivo cuyas directrices son las respuestas cognitvas a las condiciones particulares del entorno y por tanto, dan cuenta de las diferencias individuales en el funcionamiento intelectual y a la forma particular como cada uno percibe y procesa la información que el entorno le suministra. Se acepta como la expresión de las diferencias individuales la dimensión Dependencia-independencia de Campo (DIC) tal como lo propone Witkin (1985), como un rasgo característico de la personalidad del individuo que se manifiesta en sus actividades perceptivas e intelectuales entre dos extremos: el campo dependiente (CD) mas influido en su comportamiento y procesamiento de información por las referencias sociales y una percepción del entorno en forma global y el campo independiente $(\mathrm{Cl})$ por el contrario, individualista, mas confiado en sus referencias internas, analítico y con una percepción del entorno discreto y articulado.

Respaldado en lo anterior el propósito del estudio que se describe fue determinar como influye el estilo cognitivo en su dimensión DIC, en la habilidad para percibir y solucionar problemas en química. Al respecto se hicieron las siguientes suposiciones: Como los IC perciben la situación problemática en una forma discreta y articulada, dispondrán de información perceptiva mas organizada lo cual facilitará la organización de modelos mentales más apropiados y más cercanos a la interpretación científica. Por lo tanto, ante un problema dado, seleccionarán la estrategia de solución mas apropiada entre las varias disponibles que se les presenten. Los DC se verán influidos por factores de tipo perceptivo y ante las situaciones que le presenta el problema basarán su razonamiento mas en los elementos del problema directamente observables que en modelos mentales de pensamiento científico. Se espera, por consiguiente, que los primeros tengan un mejor desempeño en la solución de los problemas de química que se les propongan.

\section{ASPECTOS METODOLÓGICOS}

El estudio se realizó con 28 estudiantes del Inem de grado once, 17 de sexo masculino $(60 \%)$ y once de sexo femenino (40\%), edad promedio 17 años y en la modalidad de diversificación de ciencias y matemáticas. Se esperaba, con esta última característica de la muestra, que tuvieran semejanzas con las muestras de estudios realizados por otros grupos de investigación (Niaz, 1987) y que por esa razón pudieran ser comparables y que dadas la preferencia por las ciencias, no ofrecieran resistencia a las pruebas de solución de problema. 
La determinación de la dimensión DIC se hizo mediante la aplicación de la prueba de figuras enmascaradas (EFT) originalmente propuesta por Witkin (1991) y posteriormente adaptada por el grupo de investigación (Hederich, et al., 1994) del centro de Investigación de la Universidad Pedagógica Nacional, CIUP, a la población colombiana. Esta prueba usa la capacidad de reestructuración cognitiva como indicador para determinar la tendencia hacia la sensibilidad o hacia la independencia del medio de los sujetos sometidos a la prueba. Consta de cinco ejercicios, cada uno conteniendo una figura simple y diez complejas. El estudiante debe identificar o descubrir la figura simple enmascarada en las figuras complejas en un determinado tiempo.

Las pruebas de solución de problemas fueron problemas sencillos con temáticas que generalmente se usan en los cursos de química de bachillerato: relación peso volumen, concepto de masa, difusión de gases y ebullición de líquidos, la mayoría de ellos adaptados del trabajo de Niaz (1985). Se buscaba con estos determinar la habilidad para extraer información relevante de un texto (pregunta del problema), el grado de percepción del problema y la selección de la estrategia apropiada para llegar a la solución.

\section{RESULTADOS}

Dadas las características que exhiben los CD y los ID y las de los problemas propuestos, es evidente que del comportamiento observado en la solución de estos se puede deducir también la tendencia del estilo.

El promedio de resultados de la prueba EFT dentro de una escala de $\mathrm{O}$ a 50 puntos fue de 31,67 (DS $=7,46)$ y el $50 \%$ de la muestra $(n=14)$ obtuvieron puntajes por encima de este promedio. Esto se interpretó en el sentido de que la muestra alcanzó a resolver en promedio 32 tareas de reestructuración perceptual de las cincuenta posibles. El promedio es inusualmente alto en comparación con datos obtenidos en otros grupos, lo que permite deducir en principio que la muestra se caracteriza por exhibir un comportamiento de estilo cognitivo con mayor tendencia a la independencia de campo. El resultado sin embargo no es sorprendente, dada las características de la muestra, motivada por el estudio de las ciencias y las matemáticas, población que generalmente se identifica con tendencia de estilos cognitivos de independencia de campo.

También se deduce de los datos obtenidos ( $D S=7,46$ ), en la prueba que cerca del $46 \%$ de los puntajes se ubican entre 26 y 38 puntos, lo cual permitió interpretar que los inferiores a $26(n=8)$ pueden ser considerados sensibles o DC y los de 39 en adelante claramente IC $(n=7)$.

En el análisis de los resultados del comportamiento de los estudiantes en la solución de los problemas propuestos se centró en los claramente DC o IC. Los restantes clasificados como intermedios (IM), los cuales se supone que participan de las características de los dos estilos extremos, no se tuvieron en cuenta para el análisis y las conclusiones.

Una observación general sobre los resultados en las pruebas de solución de problemas indican que aproximadamente el $53 \%$ de la muestra tiene un comportamiento característico de los individuos IC: capacidad de reestructuración perceptiva, facilidad para extraer información de un contexto y flexibilidad para optar por procedimientos y estrategias de solución más creativas. Cuando se confrontan los estudiantes que 
observaron este comportamiento con los que obtuvieron puntaje por encima del promedio $(50 \%)$ en la prueba EFT, todos se ubican dentro de ese rango. Lo anterior además de mostrar coincidencia en los resultados de las dos pruebas, ponen en evidencia que los grupos de individuos pertenecientes a uno $u$ otro estilo, proyecta su habilidad característica en tareas de solución de problemas en Química, lo que confirma en parte los supuestos del estudio.

Un análisis mas específico en cuanto al desempeño en la solución de problemas de los estudiantes claramente definidos como IC $(n=7)$ y los claramente sensibles o $D C(n=8)$, muestra los siguientes resultados: En la prueba sobre desplazamiento de volumen y peso el 71,4\% (5/7) de los IC respondieron correctamente y los DC el 50\% (4/8). En la aprueba sobre conservación de peso el $100 \%$ de IC (7/7) se desempeñaron correctamente y el $75 \%$ de los DC (6/8)10 hicieron también. En la prueba sobre percepción de volumen el $71,4 \%(5 / 7)$ se desempeñaron correctamente y el $50 \%(4 / 8)$ DC. En la prueba sobre difusión de gases $85,7 \%$ (6/7) correctos y solo 37,5 (3/8) DC. Finalmente en el problema relacionado con el punto de ebullición y presión $62,5 \%(5 / 7)$ de IC se desempeñaron correctamente y apenas el $25 \%(2 / 8)$ de los DC.

Los resultados descritos muestra, que la DIC como dimensión del estilo cognitivo permite diferenciar en la muestra grupos de estudiantes con mayor o menor aptitud de su funcionamiento perceptivo para la solución de problemas en química. Los IC son más persistentes en la identificación y reestructuración de la información suministrada. Los DC muestran mas dificultad para aislar información relevante. Es obvio el mejor desempeño de los IC en la solución de los problemas propuestas lo cual coincide con resultados de otros estudios (Falls, 1984), quien mostró que estos son mas efectivos en la solución de problemas debido a que exhiben una mejor capacidad de razonamiento proporcional y son mas prácticos a la hora de extraer información relevante.

Los DC si bien no tan exitosos, presentan porcentajes de desempeño aceptables sobre todo en los primeros problemas, pese a las carencias observadas para la reestructuración de la información. Se infiere de este resultado y de las observaciones hechas en el proceso de solución del problema que si se dispone de mejores estrategias y procedimientos de instrucción que se adapten a las necesidades de estos diferentes tipos de estudiantes, se podría disminuir el efecto negativo de los factores perceptuales de campo en esas tareas.

Las dificultades en la solución de problemas serán siempre una realidad en el aula de clase. Si parte de estas dificultades pueden atribuirse a las características del estilo que exhiben los estudiantes en su dimensión DIC como parece resultar de las observaciones hechas en este estudio y en los otros citados, una forma de resolverlas favorablemente en el aula de clase, debe empezar por propiciar la divulgación, a los profesores de ciencias, de las características de la dimensión cognitiva y los efectos probables sobre la solución de tareas intelectuales y el establecimiento de una metodología simple que permita a los profesores identificarlas en la idea de que, con base en este conocimiento, puedan planear estrategias de instrucción mas adecuadas a las condiciones de sus estudiantes. 


\section{REFERENCIAS BIBLIOGRAFICAS}

Bodner, CM., McMillen, T. L. B. (1986). Cognitive Restructuring as an Early Stage in Problem Solving. J. of Research in Science Teaching. 23(3) pp. 727-737.

Falls,T.H., Voss, B. (1984). The ability of high school chemistry students to solve computational problems requiring proportional reasoning as affected by item task variables. Science Teaching Review of Educational Research. 94(3) pp. 63-107.

Hederich, C otros, (1994). Manual de Aplicación y Puntuación de la Prueba de Figuras Enmascaradas (EFT). Proyecto Estilos Cognitivos en Colombia. Universidad Pedagógica Nacional, CIUP.

Niaz, M. (1985). Razonamiento proporcional: Una interpretación basada en la teoría Independencia-Dependencia de Campo de Witkin. Trabajo presentado en la Primera Jornada de la Enseñanza de la Ciencia. Cumaná.

Niaz, M. (1987). Estilo Cognoscitivo y su Importancia en la Enseñanza de las Ciencias. Enseñanza de las Ciencias. 5(2) 97-104.

Witkin, H. A., Goodenough D. (1985). Estilos Cognitivos. Naturaleza y Origen. De. Pirámide S. A. Madrid.

Witkin,H. A. (otros) (1991). A Manual for the Embebed Figures Test. Palo Alto. Consulting Psychologists Press. 